\title{
Der Tumor der Papilla Vateri - eine diagnostische und therapeutische Herausforderung
}

\author{
G. Schwall und M. Trede \\ Chirurgische Klinik im Klinikum Mannheim der Universität Heidelberg, Theodor-Kutzer-Ufer, W-6800 Mannheim 1, BRD
}

In einem Beitrag aus der Chirurgischen Universitätsklinik Homburg greifen die Autoren die immer aktuelle und interessante Frage auf, inwieweit ein modifiziertes, eingeschränkt radikales Vorgehen beim Adenom des Intestinaltraktes onkologischen Prinzipien gerecht wird [9]. Bei 4 von 9 Patienten fanden auch sie ein okkultes Karzinom in einem Papillenadenom, das durch eine transduodenale Papillenexzision entfernt wurde. Eine radikale Nachoperation erfolgte nicht. Engmaschige Nachkontrollen mit Blick auf Überleben und Rezidivfreiheit bestätigen die Autoren in ihrem Vorgehen, wobei sie 5 Voraussetzungen für dieses Behandlungskonzept postulieren. Aber gerade diese 5 Punkte provozieren 5 noch nicht beantwortete Fragen:

\section{Muß beim Adenom nicht überzufällig häufig doch mit einem Karzinom gerechnet werden (sog. Adenom-Karzinom-Sequenz)?}

Bejaht man diese Frage $[2,8,10,14,17,21]$, dann ist die partielle Duodenopankreatektomie die sicherste Methode zur radikalen Entfernung des Tumors mitsamt seinen Lymphabflußbahnen $[4,5,7,18]$. Die lokale Tumorexzision gibt keine Sicherheit hinsichtlich der angestrebten $\mathrm{R}_{0}$-Resektion, zumal eine Lymphadenektomie unterbleibt.

\section{Wie zuverlässig ist die intraoperative}

Schnellschnittdiagnostik von Lymphknotenbiopsien, wenn sie schon bei der Tumordiagnostik versagt?

Gerade weil die präoperativ gewonnenen Biopsien nur bei positivem Befund verwertbar sind und allzuoft der Karzinomnachweis erst postoperativ gelingt (sog. ,okkultes Karzinom") sind Zweifel an der Zuverlässigkeit der intraoperativen Schnellschnittdiagnostik angebracht $[1,8,13,15,19]$.
3. Wie zuverlässig kann die postoperative histopathologische Diagnose nach Papillenexzision hinsichtlich des T- und N-Stadiums sein?

Da bei der lokalen Tumorexzision eine Lymphadenektomie unterbleibt, kann keine Aussage zu der Frage $\mathrm{N}_{0}$ oder $\mathrm{N}_{1}$ postoperativ getroffen werden $[3,6,16,21]$.

4. Falls es während der Nachsorge zu einem Rezidiv kommt, welche Therapiemöglichkeiten sind dann noch erfolgversprechend?

Sicher kann im Einzelfall ein Rezidiv noch durch eine Duodenopankreatektomie entfernt werden, aber häufiger gelingt der Rezidivnachweis zu spät $[1,13]$ und nur noch Palliativmaßnahmen sind möglich $[11,12]$. Ein Nachuntersuchungszeitraum von 2 Jahren erscheint angesichts der Rezidivneigung dieser Tumoren zu kurz [1, 8, $10,15]$.

5. Wie sensibel und sensitiv ist die endoluminale Sonographie, wenn es um die Frage benignes oder malignes Rezidiv geht, und diese Methode schon in der Primärdiagnostik versagt?

Der direkte Blick auf die Neopapille deckt das intraluminale Rezidiv auf. Der Nachweis eines extraluminalen Tumorwachstums mag mit Hilfe der endoluminalen Sonographie gelingen [11, 19]. Die Dignität des Befundes bleibt aber bis zur histologischen Klärung offen (siehe Frage 4).

Der Vergleich der Früh- und Spätergebnisse nach lokaler Exzision oder Duodenopankreatektomie beim Tumor der Papilla Vateri fällt in der Literatur nicht einstimmig aus. Dazu sind die Patientengruppen wohl auch zu verschieden.

Einerseits sprechen sich Autoren für die radikale Lösung (der Duodenopankreatektomie) selbst beim anscheinend benignen Adenom der Papille aus [4, 8, 19]. 
Auf der anderen Seite gibt es Verfechter der lokalen Exzision - auch beim begrenzten Karzinom [6, 12, 21].

Der in diesem Heft abgedruckte Beitrag von Koch et al. stärkt mit guten Argumenten die zweite Position.

Letztlich aber wird man dieses Problem - wie so viele in der Chirurgie - für jeden einzelnen Patienten neu diskutieren müssen.

\section{Literatur}

1. Bätz W, Bueß G, Junginger Th (1988) Adenome der Papilla Vateri. Dtsch Med Wochenschr 113:96-99

2. Baczako K, Büchler M, Beger HG, Kirkpatrick J, Haferkamp $\mathrm{O}$ (1985) Morphogenesis and possible precursor lesions of invasive carcinoma of the papilla of Vater: epithelial dysplasia and adenoma. Hum Pathol 16:305-310

3. Böttger Th, Zech J, Weber W, Soger K, Junginger Th (1989) Prognostisch relevante Faktoren bei dem Karzinom der Papilla Vateri. Langenbecks Arch Chir 374:358-362

4. Cohen JR, Kuchta N, Geller N, Shires GT, Dieneen P (1983) Pancreaticoduodenectomy for benign disease. Ann Surg 197:68-71

5. Christ DW, Sitzmann JV, Cameron JL (1987) Improved hospital morbidity, mortality and survival after the Whipple procedure. Ann Surg 206:358-365

6. Goldberg MO, Zamir O, Hadary A, Nissan S (1987) Wide local excision as an alternative treatment for periampullary carcinoma. Am J Gastroenterol 82:1169-1171

7. Grace PA, Pitt HA, Tompkins RK, den Besten L, Longmire WP (1986) Decreased morbidity and mortality after pancreatoduodenectomy. Am J Surg 151:141-149

8. Jones BA, Langer B, Taylor BR, Girotti M (1985) Periampullary tumors: which ones should be resected? Am J Surg 149:4652
9. Koch B, Hildebrandt U, Schüder G, Seitz G, Feifel G (1991) Eingeschränkte chirurgische Radikalität beim okkulten Karzinom der Papilla Vateri. Langenbecks Arch Chir 376:195-198

10. Kozuka S, Tsubone M, Yamaguchi A, Hachisuka K (1981) Adenomatous residue in cancerous papilla of Vater. Gut 22: $1031-1034$

11. Meyer J, Sulkowski U, Preusser P, Bünte H (1987) Klinik und chirurgische Therapie des Papillenkarzinoms. Zentralbl Chir $112: 20-26$

12. Neoptolemos JP, Talbot IC, Carr-Locke DC, Shaw DE, Cochleburgh R, Hall AW, Fossard DP (1987) Treatment and outcome in 52 consecutive cases of ampullary carcinoma. Br J Surg 74: $951-961$

13. Robertson JFR, Imrie CW, Hole DJ, Carter DC, Blumgart LH (1987) Management of periampullary carcinoma. Br J Surg 74: 816-819

14. Rosenberg J, Welch JP, Pyrtek JL, Walker M, Trowbridge P (1986) Beningn villous adenomas of the ampulla of Vater. Cancer 58:1563-1568

15. Rückert K, Kümmerle F (1979) Papillencarcinom ist der Tumor der periampullären Region mit der besten Prognose. Chirurg 50: $308-312$

16. Ryan DP, Shapiro RH, Warshaw AL (1986) Villous tumors of the duodenum. Ann Surg 203:301-306

17. Sellner F (1987) Untersuchung zur Hypothese der Existenz einer Adenom-Karzinom-Sequenz im Dünndarm. Z Gastroenterol 25:151-165

18. Trede M, Schwall G (1988) The complication of pancreatectomy. Ann Surg 207:39-47

19. Walsh D, Eckhauser F, Cronenwett J, Turcotte J, Lindenauer SM (1982) Adenocarcinoma of the ampulla of Vater. Ann Surg 195: $152-157$

20. Williams JA, Cubilla A, Mclean BJ, Fortner LG (1979) Twentytwo years experience with periampullary carcinoma at Memorial Sloan-Kettering Cancer Center. Am J Surg 138:662-665

21. Yamaguchi K, Munetomo E (1987) Carcinoma of the ampulla of Vater: a clinicopathologic study and pathology staging of 109 cases of carcinoma and 5 cases of adenoma. Cancer 59:506-515

Weitere Literatur bei den Verfassern 